\title{
Preparation and Properties of Blend Films Based on Poly(vinyl alcohol) and Cellulose Nanocrystals
}

\author{
Pengtao Liu ${ }^{1, a}{ }^{*}$, Weisheng Meng ${ }^{1, b}$, Shuai Wang ${ }^{1, c}$ and Yonghui Sun ${ }^{1, d}$ \\ ${ }^{1}$ Tianjin Key Laboratory of Pulp \& Paper, Tianjin University of Science and Technology, Tianjin \\ 300457, China \\ apengtaoliu@tust.edu.cn, b shengshengyi@163.com, ${ }^{c} 1542363263 @ q q . c o m$, \\ sun_yonghui00@163.com
}

Keywords: Polyvinyl alcohol; Cellulose nanocrystals; Blend film; Scanning electron microscopy; Tensile strength

\begin{abstract}
Hardwood dissolving pulp was used as the raw material for the preparation of cellulose nanocrytals $(\mathrm{CNC})$ by sulfuric acid hydrolysis. $\mathrm{CNC}$ was added into the solutions of Polyvinyl alcohol (PVA) to prepared PVA/CNC blend films. The miscibility of PVA/CNC films were studied by X-ray diffraction (XRD) and scanning electron microscopy (SEM). The strong hydrogen bonding was formed between the CNC and PVA in the mixing process, and also produced cross-linking between molecular. When the concentration of $\mathrm{CNC}$ was $1.0 \%$, tensile strength and breaking tensile strain of blend films reached the max, with the increasing amount of $\mathrm{CNC}$, flocculation of CNC was aggravated, and the compatibility of PVA and CNC was deteriorated, the mechanical properties of blend films were reduced.
\end{abstract}

\section{Introduction}

Cellulose is one of the most abundant renewable resources available today and has an annual production over $2 \times 10^{11}$ tons [1]. As one of the most common organic polymers, cellulose is considered as an almost inexhaustible source of raw material for the increasing demand for making environmentally friendly and biocompatible products. Cellulose nanocrystals (CNC) are rod-like defect free crystalline nanoparticles obtained after the acid hydrolysis of cellulose fibers, and they have special advantages including high surface areas, unique morphology, low density, and low thermal-expansion mechanical strength because of their nano scale dimensions [2-4]. These characteristics make cellulose nanocrystals suitable as nanofiller for some polymer materials.

Polyvinyl alcohol (PVA) is an important water soluble polymer, and it has been widely used in medicine, construction, wood processing, paper making, printing, agriculture, polymer chemical industry because of its excellent film forming, emulsifying and adhesive properties [5-7]. The improvement of PVA's mechanical properties is valuable.

PVA and cellulose are polar polymers; thus a composite of PVA and cellulose is likely to produce a material having excellent mechanical properties. Incorporation of cellulose into the PVA matrix changes the physicochemical properties of the material and thus modifies the polymer structure at both the molecular and the morphological levels. This study reports on the effect of CNC content on the properties of PVA films, such as mechanical and microstructural parameters. These studies were carried out to assess their potential uses as films.

\section{Materials and Methods}

Materials. Hardwood dissolving pulp, with white degree of $87 \% \sim 89 \%$ ISO, moisture content of $5 \% \sim 6 \%$, the content of $\alpha$-cellulose of over 95\%, the fiber length of $0.6 \sim 0.7 \mathrm{~mm}$ and the average degree of polymerization is about 700, was obtained from Shandong Pulping and Paper co., Ltd and was used as raw materials. Polyvinyl alcohol(PVA) was supplied by Beijing Entrepreneur Science \& 
Trade Co., Ltd., the average degree of polymerization is about 1700 , and the alcoholysis degree is $88 \%$. The other chemical reagents were obtained from commercial resources in China and used as received.

Preparation and Characterization of CNC [8,9]. The suspensions of CNC were prepared by hydrolysis under $60 \% \mathrm{w} / \mathrm{w}$ sulfuric acid. Typically, $10 \mathrm{~g}$ dry hardwood dissolving pulp was treated with $167 \mathrm{~g}$ sulfuric acid at $35^{\circ} \mathrm{C}$ for $3.5 \mathrm{~h}$ with constant stirring, and then the suspension was diluted with purified water to stop the reaction. The suspension was centrifuged at $8000 \mathrm{rpm}$ for 10 minutes to concentrate suspensions and to remove excess water and acid for three times. The precipitate was adjusted to neutral with sodium hydroxide, and was dialyzed against water for 7 days. Finally, the fluorescent blue suspensions were got after ultrasonic processing. Laser particle analyzer (90plus Brookharen Corporation USA) and Atom force microscopy (JSPM-5200, JEOL, Japan) were used to detected the size of the CNC.

Preparation and Characterization of Blend Films. PVA was mixed with tween-80 in a ratio of 9:1, and different amount of CNC was added. The mixtures were stirred at $90{ }^{\circ} \mathrm{C}$ for $20 \mathrm{~min}$, and clear and transparent solution was formed. A certain amount of ethanol was added in the system after the blend solution was standing for $5 \mathrm{~min}$, and the system was stirred uniformly. Before casting, the mixtures was filtered, the thickness of the film was controlled by pouring a known weight of the blend solution into a flat-bottomed glass disc[10]. The blend film was dried in vacuum oven at $40{ }^{\circ} \mathrm{C}$ for $48 \mathrm{~h}$.

The blend films with different contents of PVA and CNC were frozen in liquid $N_{2}$, immediately fractured, sputtered with gold and photographed. The surface and cross-section of films were carried out on scanning electron microscopy (JSM-6380LV JBOL Japan) with an acceleration voltage of 15 $\mathrm{kV}$.

X-ray diffraction (XRD) measurements of the blend films were carried out on a X-ray diffractometer (TD-3500 Tongda China) equipped with a $\mathrm{Cu} \mathrm{K \alpha}$ radiation source. The suitable shape of blend films were placed in samples groove, the test conditions were $30 \mathrm{kV}$ of tube voltage, $20 \mathrm{~mA}$ of tube current, $2 \theta=10 \sim 30^{\circ}$ of scanning range and $4.8^{\circ} / \mathrm{min}$ of scanning speed.

Electronic universal tensile machine (WDW Songdun China) was used to detect the tensile strength and breaking tensile strain of blend film, referring to the GB1040-1992. The blend films were cut into fix-size strips for detection, and each sample was measured 5 times repeatedly for the average.

\section{Results and Discussion}
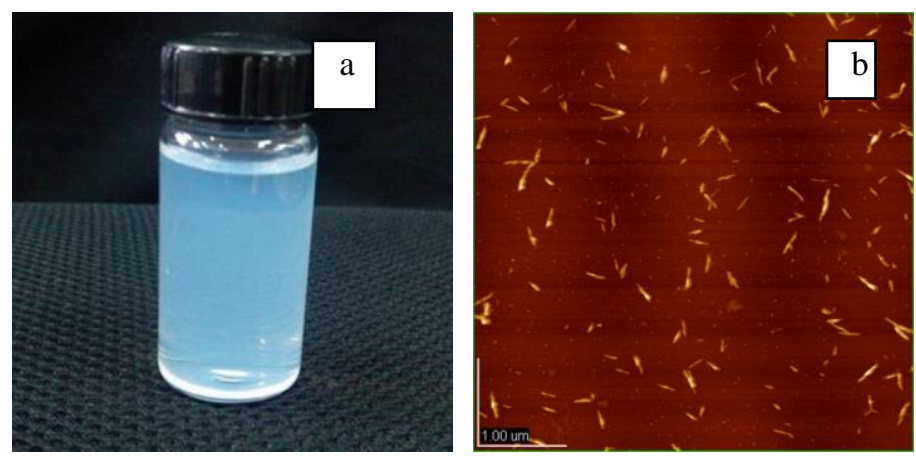

Figure. 1 Suspension and AFM image of CNC

Characterization of CNC. Fig. 1a is the CNC suspension which was prepared by the sulfuric acid hydrolysis method. The suspension was fluorescent blue translucent liquid. Fig. 1b shows the AFM images of CNC. It was found that CNC was rod-like structure with 200-300nm long and 10-30nm wide, which were all in nanometer range. The mean length of CNC was approximately $260 \mathrm{~nm}$ detected by Laser particle analyzer, which was identical to the results of AFM.

SEM Observations. Fig. 2 shows the SEM images of surface of blend films, and the concentration of $\mathrm{CNC}$ from a to e were $0,0.5 \%, 1.0 \%, 1.2 \%$, and $1.5 \%$ respectively. As can be seen from figures, the 
surfaces of blend films were smooth when the concentrations of CNC were less than $1.0 \%$ in Fig. 2a-b. While the contents of CNC were exceed $1.0 \%$, some white crystalline substances were produced, and the surfaces of blend films were more uneven and rougher in Fig. 2c-e. This suggested that CNC and PVA have a better compatibility when the amount of CNC was less, but with the increasing contents of $\mathrm{CNC}$, the flocculation of $\mathrm{CNC}$ were more and more seriously, the compatibility of the two points became worse, and the homogeneity and surface smoothness of PVA/CNC blend films were reduced significantly.

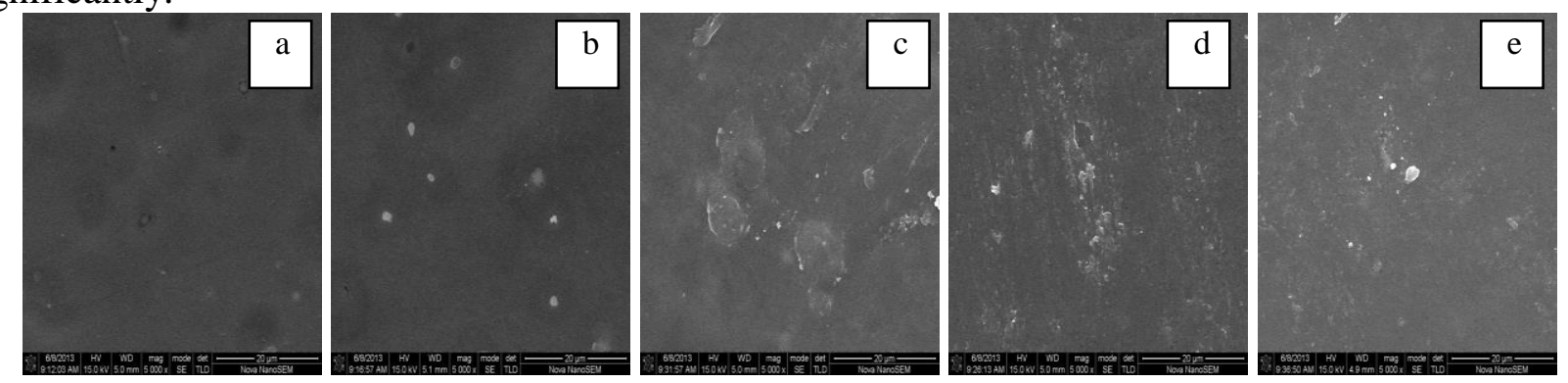

Figure. 2 SEM images of surface of blend films with different CNC contents (the concentration of

$\mathrm{CNC}$ from a to e were $0,0.5 \%, 1.0 \%, 1.2 \%$, and $1.5 \%$ respectively, $\times 5000$ )

Fig. 3 shows the SEM images of cross-section of blend films with different CNC contents, and the concentration of $\mathrm{CNC}$ from a to e were $0,0.5 \%, 1.0 \%, 1.2 \%$, and $1.5 \%$ respectively. The cross-section of pure PVA film was loose, and some obvious interspaces appeared in Fig. 3a. Compared with the film without adding CNC, the cross-section of PVA/CNC blend films were more dense relatively in Fig.3b-d, and with the increasing content of $\mathrm{CNC}$, the white crystalline substances became more and more apparent. So CNC had some impacts on the structure of blend films, it was because the hydroxyl groups on the surfaces of PVA and CNC can form hydrogen bonds, and generate cross-linking. The distance between the molecules of films was reduced within the strong interaction, and the structure of film became closer. The white crystalline substances in the cross-section of blend films were the same as the surfaces ones, it also caused by flocculation of $\mathrm{CNC}$.
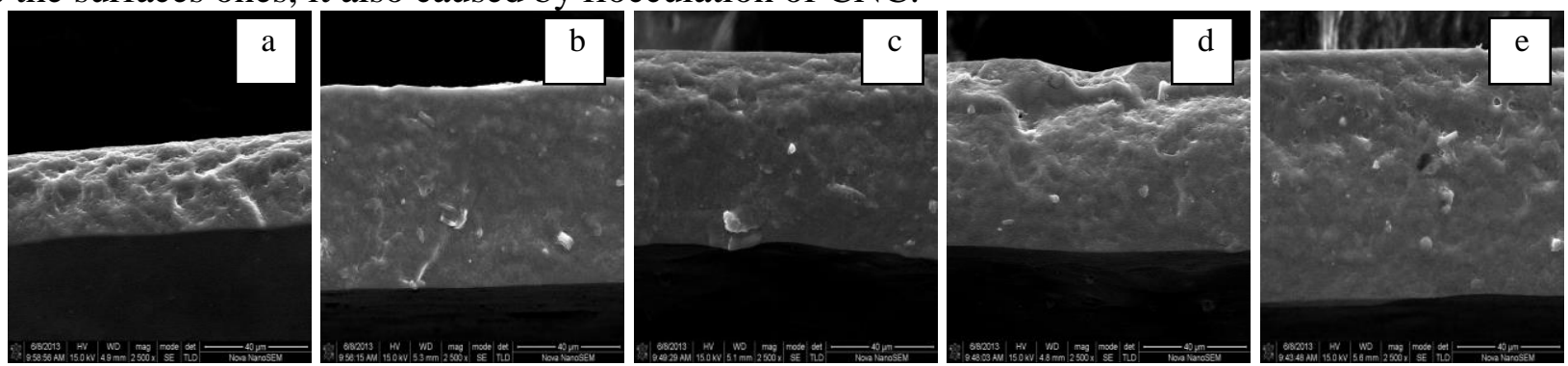

Figure. 3 SEM images of cross-section of blend films with different CNC contents (the concentration of CNC from a to e were $0,0.5 \%, 1.0 \%, 1.2 \%$, and $1.5 \%$ respectively, $\times 2500$ )

XRD Analysis. Fig. 4 is the XRD spectra of mixed films. It can be seen from Fig. 4a, pure PVA film had one absorption peak at $19.3^{\circ}$, it was because that the hydroxyl groups on the surface of PVA have small volume and strong polarity, and being arranged highly ordered crystal lattice easily. The $2 \theta$ of cellulose were $14.8^{\circ}, 16.5^{\circ}$ and $22.7^{\circ}$ respectively, corresponding to $\{101\},\{10 \overline{1}\}$ and $\{002\}$ planes of cellulose crystalline. Compared with the diffraction peak intensity of $22.7^{\circ}$, the diffraction peak at $14.8^{\circ}$ and $16.5^{\circ}$ were much weaker, and the content of $\mathrm{CNC}$ was low relatively, therefore the two diffraction peaks were not reflected in Fig. 4. As the result of the addition of CNC to PVA, a diffraction peak at about $23^{\circ}$ was appeared and increasingly strength, the crystallinity of blend films were also increased. The $2 \theta$ of diffraction peaks at $19.3^{\circ}$ had a small offsets with the addition of CNC, it means that the spacing of blend films crystal planes were smaller than pure PVA films', and this results was consistent with the measurement of SEM on the cross-section of blend films. CNC played a role of a nucleating agent in the process of mixing, the two components were produced interaction, and limited the movement of molecular chains, promoted the formation of crystal structure, resulted in the spacing 
of PVA crystal planes reduced, and the crystallinity of blend film increased and the structure of blend films became denser.

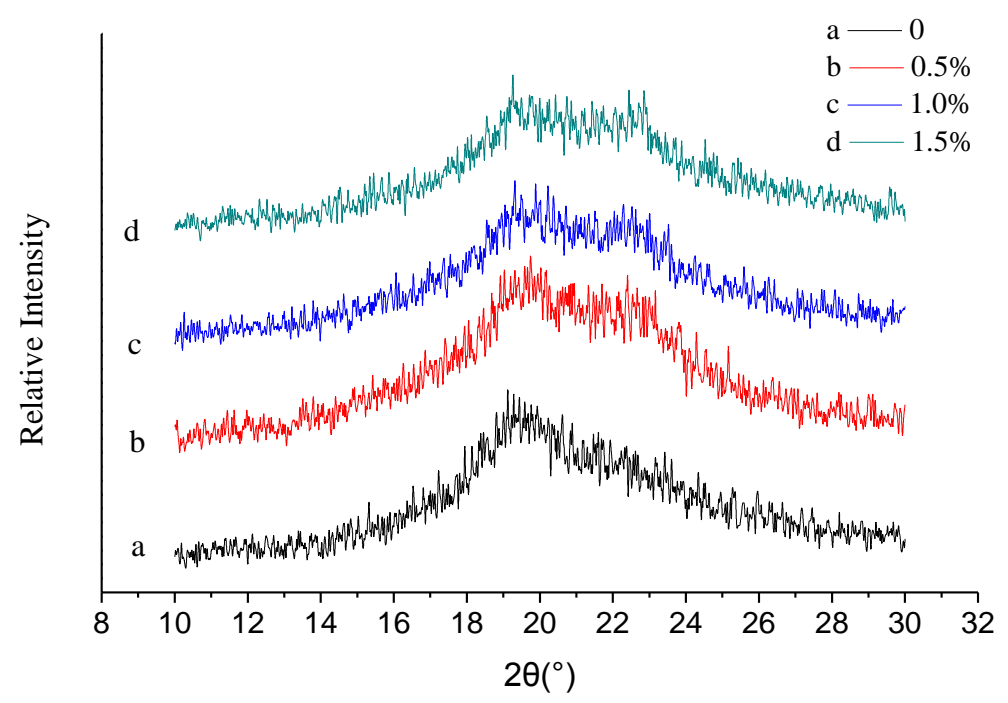

Figure. 4 XRD patterns of mixed films with different CNC contents (the concentration of CNC from a to $\mathrm{d}$ were $0,0.5 \%, 1.0 \%$, and $1.5 \%$ respectively)

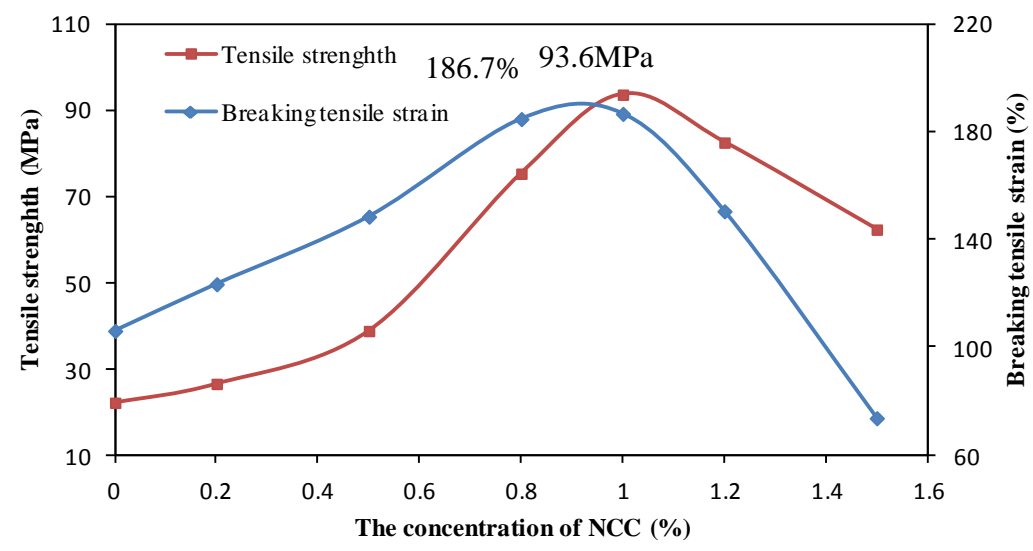

Figure. 5 Effect of the concentration of $\mathrm{CNC}$ on the tensile strength and the breaking tensile strain of blend films

Mechanical Properties. Tensile strength and breaking tensile strain were performed in order to evaluate the effect of CNC on the mechanical properties of blend films. It can be seen from Fig. 5, there was no linear change about tensile strength and the breaking tensile strain on the concentration of CNC in blend films, the two curves were first increase and then decrease, and all reached max at about $1.0 \%$ of CNC concentrations, the value were $93.57 \mathrm{MPa}$ and $186.68 \%$ respectively. Compared with pure PVA film which the tensile strength and breaking tensile strain were $22.1 \mathrm{MPa}$ and $186.68 \%$, they were increased by $324.5 \%$ and $76.1 \%$ respectively at about $1.0 \%$ of CNC concentrations. This results further illustrates that the complex process of PVA and CNC was not a simple two-component mixing, some interactions exited between two points. With the increasing of $\mathrm{CNC}$, the amount of hydrogen bonds were also increased between PVA and CNC, and when the concentration of CNC was at about $1.0 \%$, two components produced a better synergy, mechanical properties of blend films were best. While with the further increased of $\mathrm{CNC}$, flocculation of $\mathrm{CNC}$ was aggravated and the compatibility became poor, tensile strength and breaking tensile strain decreased. It is worth noting that when the concentration was $1.5 \%$, tensile strength was still bigger than pure film ones, but breaking tensile strain was smaller than the pure film ones, this is because that three-dimensional network structure were formed during 
the composite process of PVA and $\mathrm{CNC}$, it caused the deterioration of extending performance on single level, and made the tensile strain of blend film on single direction also reduced.

\section{Conclusions}

The strong hydrogen bonding was formed between the CNC and PVA in the mixing process, and also produced cross-linking between molecular. When the concentration of $\mathrm{CNC}$ was $1.0 \%$, tensile strength and breaking tensile strain of blend films reached the max, with the increasing amount of CNC, flocculation of CNC was aggravated, and the compatibility of PVA and CNC was deteriorated, the mechanical properties of blend films were reduced. $\mathrm{CNC}$ had a promoting role on the nucleation of PVA matrix, and also had a certain influence on crystal structure during the formation process of blend films, the spacing of PVA crystal planes was reduced, then crystallinity were increased, and the blend films became denser.

\section{Acknowledgements}

The financial support for this project was from the Tianjin Municipal Science and Technology Commission (Grant No.12ZCZDGX01100), the Scientific Research Foundation of the State Key Laboratory of Pulp and Paper Engineering (No.201119), and the Foundation of Tianjin Key Laboratory of Pulp \& Paper (Tianjin University of Science \& Technology, No.201313), P. R. China.

\section{References}

[1] W. Bai, J. Holbery and K.C. Li: Cellulose, Vol. 16 (2009) No.3, p.455.

[2] J.S. Fan and Y.H. Li: Carbohydrate Polymers, Vol. 88 (2012) No.4, p.1184.

[3] W. Li, J.Q. Yue and S.X. Liu: Ultrasonics Sonochemistry, Vol. 19 (2012) No.3, p. 479.

[4] E. Lam, K.B. Male, J.H. Chong, A.C.W. Leung and J.H.T: Trends in Biotechnology, Vol. 30 (2012) No.5, p. 283.

[5] S.A. Li, Y. Gao, H.L. Bai, L.P. Zhang, P. Qu and L. Bai: Bioresources. Vol. 6 (2011) No.2, p. 1670.

[6] S. Virtanen, S. Vuoti, H. Heikkinen and P. Lahtinen: Cellulose, Vol. 21 (2014) No.2, p. 3561.

[7] X. Xu, Y.Q. Yang, Y.Y. Xing, J.F. Yang and S.F. Wang: Carbohydrate Polymers, Vol. 98 (2013) No.6, p. 1573.

[8] M. Donald and A. H. Windle: Liquid Crystalline Polymers (Cambridge University Press, England 1992), p. 13.

[9] C. Y. Bao, D. R. Long and C. Vergelati: Carbohydrate Polymers, Vol. 116 (2015) No.6, p. 95.

[10]A.M. Torres-Huerta, D. Palma-Ramrez, M.A. Domnguez-Crespo, D. Del Angel-Lpez, and D. De La Fuente: European Polymer Journal, Vol. 64 (2014) No.6, p. 285. 